Article

\title{
Novel Luminescent Multilayer Films \\ Containing $\pi$-Conjugated Anionic Polymer with Electronic Microenvironment
}

\author{
Tianlei Wang, Meitang Liu * and Hongwen Ma \\ Beijing Key Laboratory of Materials Utilization of Nonmetallic Minerals and Solid Wastes, \\ National Laboratory of Mineral Materials, School of Materials Science and Technology, \\ China University of Geosciences, Beijing 100083, China; wangtl@cugb.edu.cn (T.W.); \\ mahw@cugb.edu.cn (H.M.) \\ * Correspondence: mtliu@cugb.edu.cn; Tel.: +86-10-8232-0151 \\ Academic Editor: Giorgio Biasiol \\ Received: 1 August 2016; Accepted: 19 September 2016; Published: 27 September 2016
}

\begin{abstract}
Layered double hydroxides (LDHs), luminescent $\pi$-conjugated anionic polymer and montmorillonite (MMT) were orderly assembled into luminescent multilayer films via layer-by-layer self-assembly method. The electronic microenvironment (EME), the structure of which is like a traditional capacitor, can be constructed by exfoliated LDHs or MMT nanosheets. In addition, the rigid inorganic laminated configuration can offer stable surroundings between the interlayers. As a result, we conclude that EME can extend the luminescent lifespans of multilayer films substantially, due to affecting relaxation times of $\pi$-conjugated anionic polymer. Consequently, because of the remarkable impact on better photoemission behaviors of luminescent $\pi$-conjugated anionic polymer, EME assembled by LDHs or MMT nanosheets have had high hopes attached to them. They are expected to have the potential for designing, constructing, and investigating novel light-emitting thin films.
\end{abstract}

Keywords: thin films; luminescent; multilayers

\section{Introduction}

Nowadays, layered materials, due to their unique nanostructure and specific low-dimensional qualities, have been widely used for designing, constructing and investigating novel ordered layer-structure functional materials [1-4]. Kotov et al. successfully fabricated stiff and ultrastrong nanocomposites by assembling alternately with montmorillonite (MMT) nanosheets and polyvinyl alcohol (PVA) aqueous solution, which upsurges a target for preparation nanocomposite materials via layer-by-layer self-assembly method (LBL method) [5-7]. As successful exfoliation of layered double hydroxides (LDHs), positively-charged LDHs nanosheets have been widely used as building blocks [8-15].

Recently, our group has successfully verified that an electronic microenvironment (EME) can enhance the multilayer films' lifetimes containing cationic chromophores about 40-fold as long as those of the original powders [16]. Exfoliated LDHs and MMT nanosheets constructed a nano-system via LBL method, and then EME can be formed by the oppositely-charged rigid inorganic nanosheets between the interlayers, as shown in Figure 1. The nano-system structure is like a traditional capacitor's structure, in which the positive LDHs nanosheets are used as positive plates of capacitors and negative MMT nanosheets are as negative plates of capacitors. Between the interlayers, the EME can be provided. At the same time, we also confirm that the ferromagnetic effect, which is formed by transition metal-bearing LDHs nanosheets, is fairly conducive to enhancing the lifetimes of multilayer films, and better photoemission behavior is obtained thanked to the double coupling effects of EME 
and magnetic field $[17,18]$. However, the novel concept has only been clarified in several nano-systems, but the questions of whether EME's effect is endowed with universality for all chromophores, and whether it can also be solely beneficial to the lifetimes of multilayer films containing the anionic luminescent polymer except nanosheets' ferromagnetic effect, need to be clearly explored imminently.

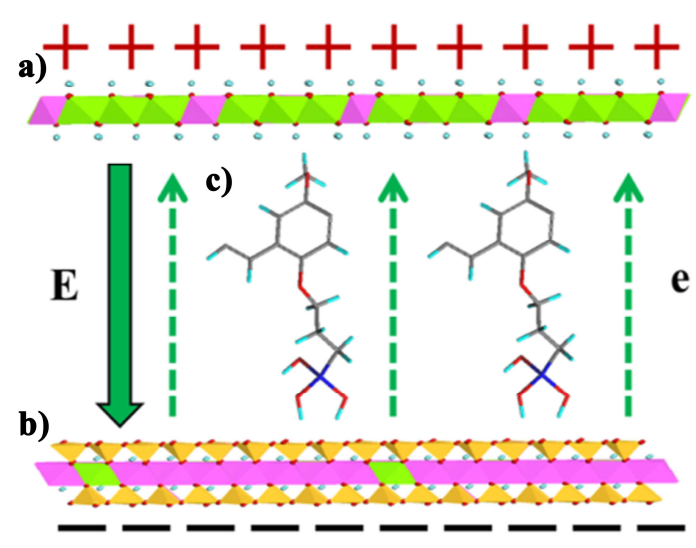

Figure 1. The sketch map of APPV in electronic microenvironment (EME) between the montmorillonite (MMT) and layered double hydroxides (LDHs) monolayers. (a) MgAl-LDHs monolayer; (b) MMT monolayer; (c) Poly [5-methoxy-2-(3-sulfopropoxy)-1,4-phenylenevinylene] (APPV). In this figure, the pink stands for aluminum element, green stands for magnesium element, yellow stands for silicon element.

In this work, anionic luminescent $\pi$-conjugated polymer was intercalated into the different inorganic nanosheets for fabricating multilayer films, in which optically-inert PVA was used as bonder. A preconceived nano-scaled architecture assembled by oppositely-charged nanosheets can form EME between the interlayer, and then the multilayer films exhibit remarkable luminescent properties with ultralong lifespans. This work provides fascinating possibilities for the development of the next generation of optoelectrical, optomagnetic devices based on the inorganic oppositely-charged nanosheets.

\section{Materials and Methods}

Poly [5-methoxy-2-(3-sulfopropoxy)-1,4-phenylenevinylene] (APPV, 0.25 wt \% in $\mathrm{H}_{2} \mathrm{O}$ ) was provided by Sigma Chemical Co. Ltd. (Beijing, China) $\mathrm{Mg}\left(\mathrm{NO}_{3}\right)_{2} \cdot 6 \mathrm{H}_{2} \mathrm{O}, \mathrm{Al}\left(\mathrm{NO}_{3}\right)_{3} \cdot 9 \mathrm{H}_{2} \mathrm{O}$ were supplied by Sinopharm Chemical Reagent Co. Ltd. (Shanghai, China) $\mathrm{NaOH}, \mathrm{H}_{2} \mathrm{O}_{2}(30 \%), \mathrm{H}_{2} \mathrm{SO}_{4}$ (95\%-98\%) were provided by Beijing Chemical Reagent Company (Beijing, China). Na-montmorillonite (MMT) was manufactured by Zhejiang Feng Hong New Materials Co. Ltd. (Huzhou, China), and polyvinyl alcohol (PVA, DP = $1750 \pm 50$ ) was supplied from Tianjin Fuchen Chemical Reagent Plant (Tianjin, China).

UV-vis absorption spectra were recorded by double beam Persee UV-vis spectrophotometer (TU-1901, Beijing, China). Fluorescence spectra was performed on a fluorescence spectrophotometer (F-4600, Hitachi, Tokyo, Japan). X-ray diffraction pattern was recorded by Rigaku X-ray Diffractometer (XRD, RIGAKU D/Max-2550 with Cu K $\alpha$ radiation, Tokyo, Japan) under the conditions: $40 \mathrm{kV}, 30 \mathrm{~mA}$. The morphology of the sample was investigated by Hitachi S-4300 Scanning Electron Microscope (SEM, Tokyo, Japan). The fluorescence decay was tested by using Edinburgh Instruments' steady and transient time-resolved fluorescence spectrometer, and the $375 \mathrm{~nm}$ pulse laser radiation was used as the excitation source (FLS-920, Livingston, UK).

An aqueous mixed nitrate solution $(50 \mathrm{~mL})$ dissolved $\mathrm{Mg}\left(\mathrm{NO}_{3}\right)_{2} \cdot 6 \mathrm{H}_{2} \mathrm{O}(0.4 \mathrm{~mol}), \mathrm{Al}\left(\mathrm{NO}_{3}\right)_{3} \cdot 9 \mathrm{H}_{2} \mathrm{O}$ $(0.2 \mathrm{~mol})$ was prepared, and then mixed with the solution of $\mathrm{NaOH}(50 \mathrm{~mL}, 2.4 \mathrm{~mol} / \mathrm{L})$ with vigorous stirring at $80{ }^{\circ} \mathrm{C}$ for $24 \mathrm{~h}$ under $\mathrm{N}_{2}$ gas, and then solid were centrifuged and dried at $60^{\circ} \mathrm{C}$. 
$0.1 \mathrm{~g} \mathrm{MgAl-LDHs}$ was mixed with $100 \mathrm{~mL}$ formamide, and flake by a mechanical shaker for 2 days. One gram of MMT was added to $1 \mathrm{~L}$ deionized water an unremittingly stirred for 28 days. Then, centrifuge this turbid liquid at 10,000 rpm for $10 \mathrm{~min}$ in order to obtain a solution with exfoliated MMT nanosheets. Quartz slides were cleaned in $\mathrm{H}_{2} \mathrm{SO}_{4}$ and $\mathrm{H}_{2} \mathrm{O}_{2}$ solution for $30 \mathrm{~min}$. PVA aqueous solution $(1 \mathrm{wt} \%)$ acted as the bonder to connect the different components, and then $0.025 \mathrm{wt} \%$ APPV aqueous solution was mixed with isopyknic PVA aqueous solution to form APPV@PVA solution. (MMT/APPV@PVA/MgAl-LDHs/APPV@PVA) $n$ (marked as MALA(n)-films) were obtained by repeating as follow: (i) steeping the substrate into MMT solution for $5 \mathrm{~min}$, and then thoroughly cleaning it; (ii) steeping it into APPV@PVA solution for $5 \mathrm{~min}$, and cleaning it; (iii) steeping it into MgAl-LDHs suspension for $5 \mathrm{~min}$, and cleaning it; (iv) steeping it into APPV@PVA solution for 5 min,

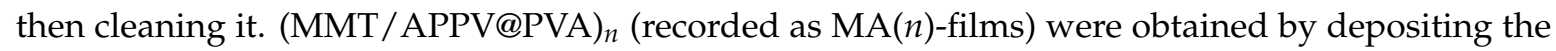
MMT suspension, APPV@PVA solution in turn for each 5 min, respectively.

\section{Results}

Figure 2a shows the UV-vis absorption spectra and photoluminescence spectra of MA $(n)$-films with varying numbers of layers $(n=4,8,12,16,24,32)$, and the inset of left figure indicates the intensities of absorption peaks increase linearly at $293.5 \mathrm{~nm}$ and $452.5 \mathrm{~nm}$ when the layers grow, displaying a well-organized growth in assembly process of those thin films. Under $375 \mathrm{~nm}$ excitation, these thin films give a green emission band peak at $572.0 \mathrm{~nm}$ (Figure 2b). Meanwhile, the intensities of fluorescence peaks also increase gradually as the deposition cycles increase, which shows that the films grow stepwise. Moreover, as shown in Figure $2 \mathrm{c}$ about small angle XRD peaks, the intensities of multilayer films increase with the deposition cycles, and the films have well-organized structure in the vertical direction with a period of ca. $4.18 \mathrm{~nm}$. At the same time, $\mathrm{MA}(n)$-films are extremely regular and smooth in the SEM image (Figure 2d). Surprisingly, the luminescent lifetimes of MA $(n)$-films range from $1.32 \mathrm{~ns}$ to $1.41 \mathrm{~ns}$, nearly 2.2 times as long as poly [5-methoxy-2-(3-sulfopropoxy)-1,4-phenylenevinylene] (APPV) solution (0.6 ns), about 1.0 times longer than those of (LDHs/APPV) ${ }_{n}{ }^{\prime}$ (marked as LA( $n$ )-films, 0.66-0.81 ns) [15]. Negative-charged MMT nanosheets can provide isolation effect and electrostatic repulsion for the anionic APPV, thus leading to the APPV stacking more orderly and suppressing the formation of aggregates.

In order to confirm EME's effect, referred to in our present work on the anionic chromophores, $\operatorname{MALA}(n)$-films were fabricated via a similar method to the present work [15]. Figure 3a shows the UV-vis absorption spectra of MALA $(n)$-films, which grow in a stable step with the layer number. The peak of absorption at $293.5 \mathrm{~nm}$ and $452.5 \mathrm{~nm}$ are absorption of phenylene ring and $\pi-\pi^{*}$ transition of APPV. The emission band peak is seen at $578.0 \mathrm{~nm}$ as yellow in color, and the emission intensity also increases consistently with the layer number (Figure 3b). Compared to the MA $(n)$-films, the luminescent peak occurs during a red-shift. When introducing the rigid oppositely-charged inorganic nanosheets, isolation effect and EME can affect either intermolecular interaction or entangled main-chain. Firstly, they can have an impact on intramolecular phenyl-phenyl stack structure, which give rise to emerge a decrease in electronic $\pi^{*}-\pi$ transition energy (red shift in luminescent band) and the increase in oscillator strength. Secondly, they can also make the entangled main-chain loosen and flatten. As a result, energy-trapping sites with lower electronic transition energies are more easily formed. Moreover, small angle XRD and SEM images show that multilayer films are regular and uniform with a period of ca. $6.74 \mathrm{~nm}$ (Figure 3c,d). Importantly, the lifetimes of MALA(n)-films (1.47-1.59 ns) are about 1.50 times longer than APPV solution $(0.60 \mathrm{~ns})$, are also prolonged to nearly 2.0-fold combined with LA(n)-films' (0.66-0.81 ns), and with an increasing than MA(n)-films (1.32-1.41 ns). The dominant factor is that LDHs and MMT inorganic nonosheets can assemble EME in this nano-architecture, which can affect the vibration of backbone and extend the relaxation time, finally prolonging lifetimes $[19,20]$. Above all, EME is helpful to improve photoemission behaviors of multilayer films. When introducing MgAl-LDHs and MMT nanosheets into the nano-system, lifetimes of chromophores can be prolonged by a large margin. 
The quantum yield (QY) of MALA $(n)$-films $(n=8,16,24)$ are $0.001363 \%, 0.001319 \%, 0.001598 \%$, and the QY of MA(n)-films $(n=8,16,24)$ are $0.001990 \%, 0.002032 \%, 0.002143 \%$. The first reason for the so low QY values is the imbalance of hole/electron injection and transport because the emissive polymer conduct holes are used in preference to electrons [21,22]. Due to the easily trapped excitons and constant nonradiative decay, there is the presence of defects in the polymer, so this is the second reason $[23,24]$. The third reason for the low $Q Y$ is because of interchain interactions such as aggregation and excimer formation, leading to a quenching process when adding the polyvinyl alcohol as a binder $[25,26]$. Finally, we introduce the negative nanosheets which repulse the anionic APPV, leading to a reduction in the concentration of APPV, which affects the weaker intensity of luminescence and the lower QY.
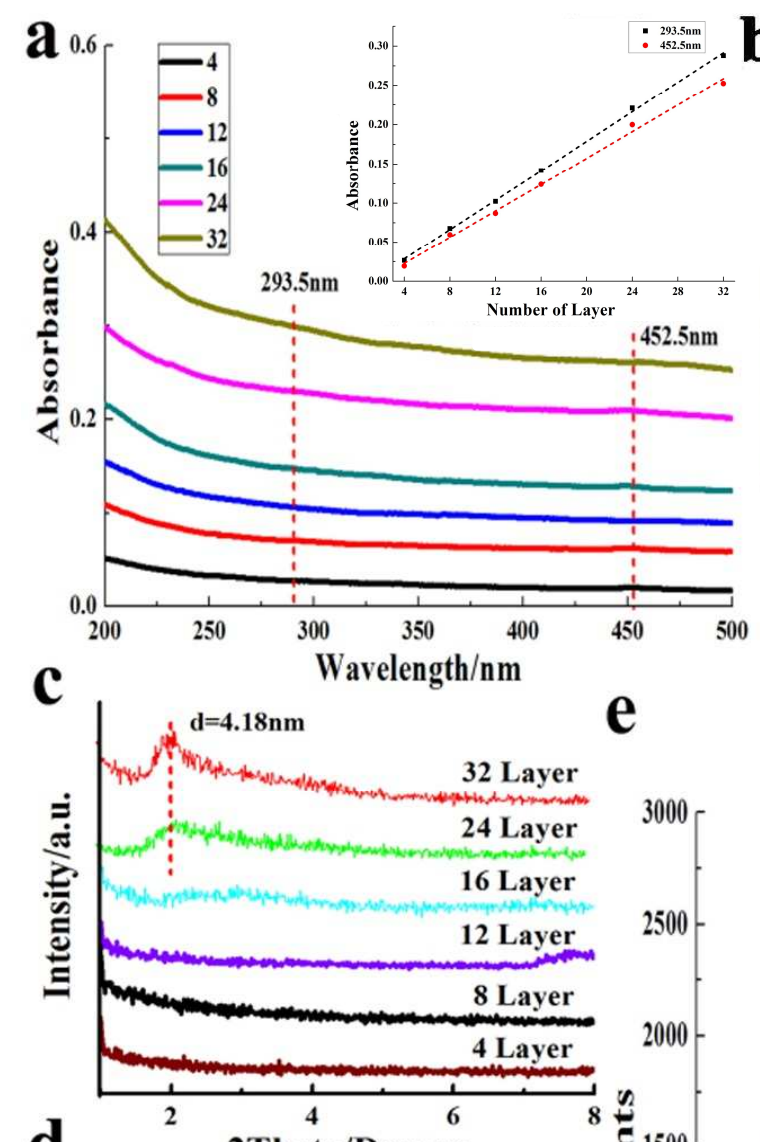

e
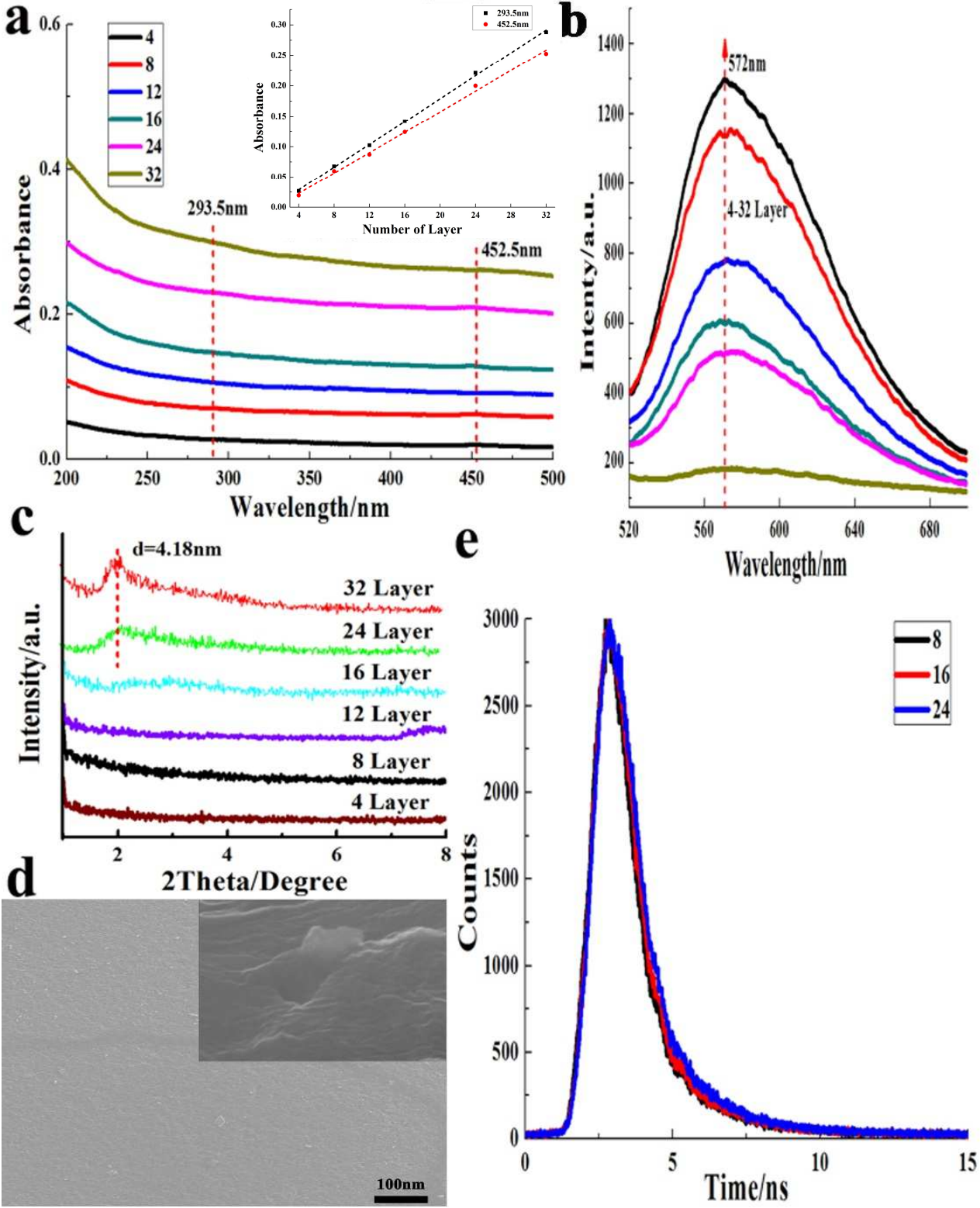

Figure 2. Characterization of (MMT/APPV@PVA) $n$ (MA(n) films) (a) UV-vis absorption spectra (the inset shows the absorbance at $293.5 \mathrm{~nm}$ and $452.5 \mathrm{~nm}$ increasing linear relationship; (b) fluorescence spectra; (c) Small angle X-ray Diffractometer (XRD) patterns; (d) Scanning Electron Microscope (SEM) images of the thin films surface, and the inset show the thin films' cross sections; (e) Fluorescence decay profiles. 

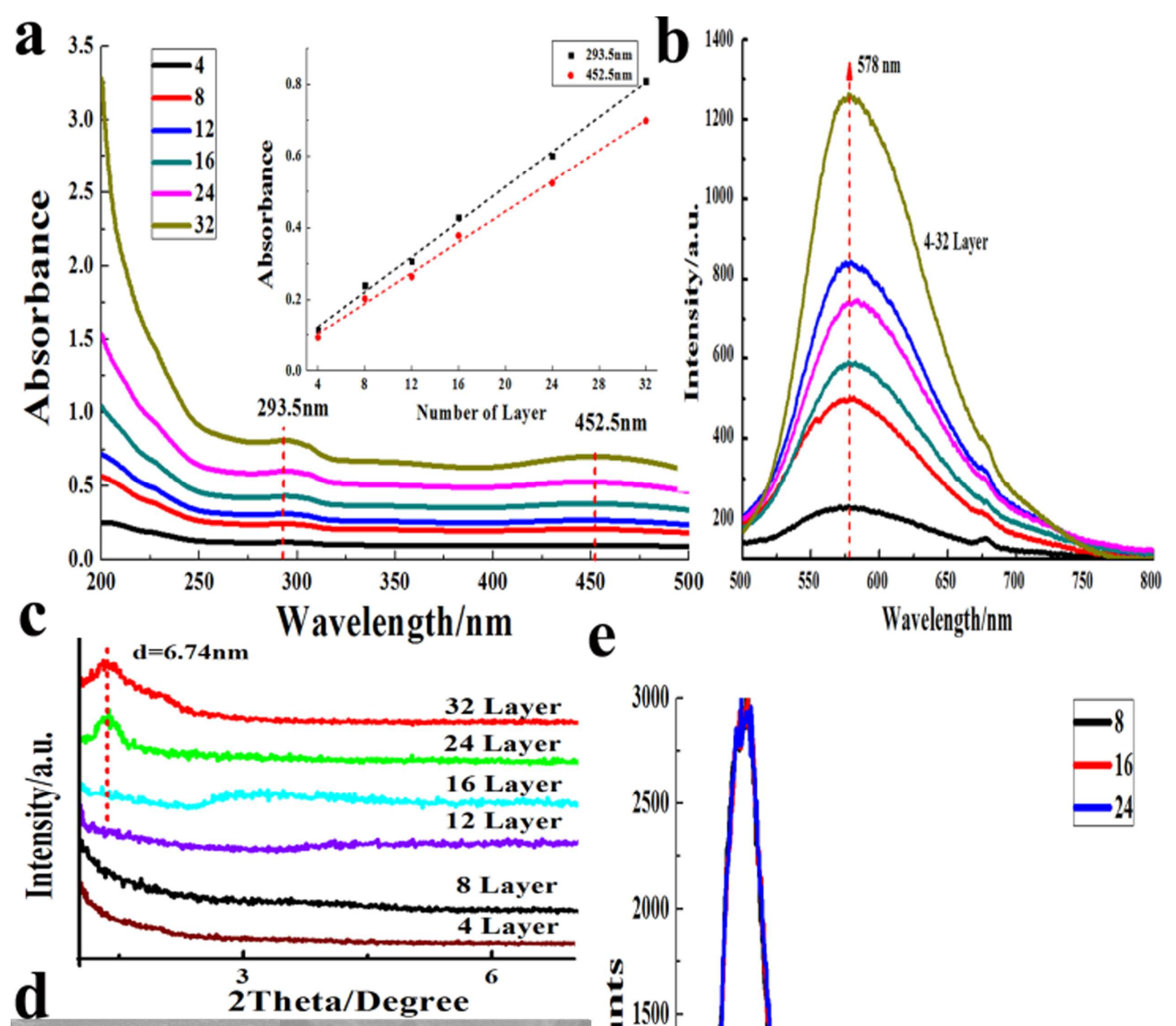

$100 \mathrm{~nm}$

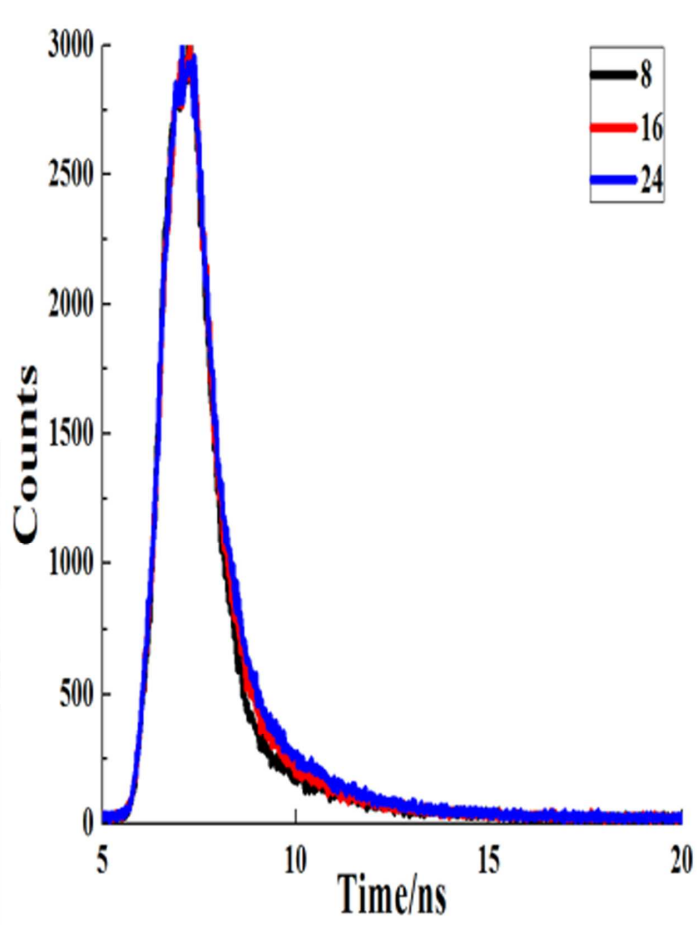

Figure 3. Characterization of (MMT/APPV@PVA/MgAl-LDHs/APPV@PVA) $n$ (MALA(n) films). (a) UV-vis absorption spectra (the inset shows the absorbance at $293.5 \mathrm{~nm}$ and $452.5 \mathrm{~nm}$ increasing linear relationship; (b) fluorescence spectra; (c) Small angle XRD patterns; (d) SEM images of the thin films surface, and the inset show the thin films' cross sections; (e) Fluorescence decay profiles.

\section{Discussion}

A battery of novel luminescent thin films containing anionic APPV based on MgAl-LDHs and MMT nanosheets were successfully assembled via LBL method. Significantly, APPV were intercalated into EME which was built by oppositely-charged inorganic layer, and EME, which can affect the vibration of backbone and extend the relaxation time, was verified to process the ability to enhance the lifetimes.Figure 4 illustrates the comparison of APPV's luminescent lifetimes under different microenvironments, and the black, red, blue and bottle green dots stand for the lifetimes of APPV, 
LA(n)-films, MA(n)-films and MALA(n)-films, respectively. Due to the rigid structure of MgAl-LDHs, the headmost step is formed. In the nano-system, rigid inorganic nanosshets can promote APPV dispersion better and eliminate their stacking interaction. The second step is due to MMT nanosheets' ability to provide isolation effect and electrostatic repulsion for the anionic APPV, thus leading to the APPV stacking in a more orderly way and suppressing the formation of aggregates. The crucial reason for the last noteworthy step is that EME can be formed by oppositely-charged inorganic nanosheets, which can definitely affect relaxation times of $\pi$-conjugated anionic polymer. Above all, it is obviously confirmed that EME in the nano-system can be beneficial for the lifetimes of chromophores between the interlayers.

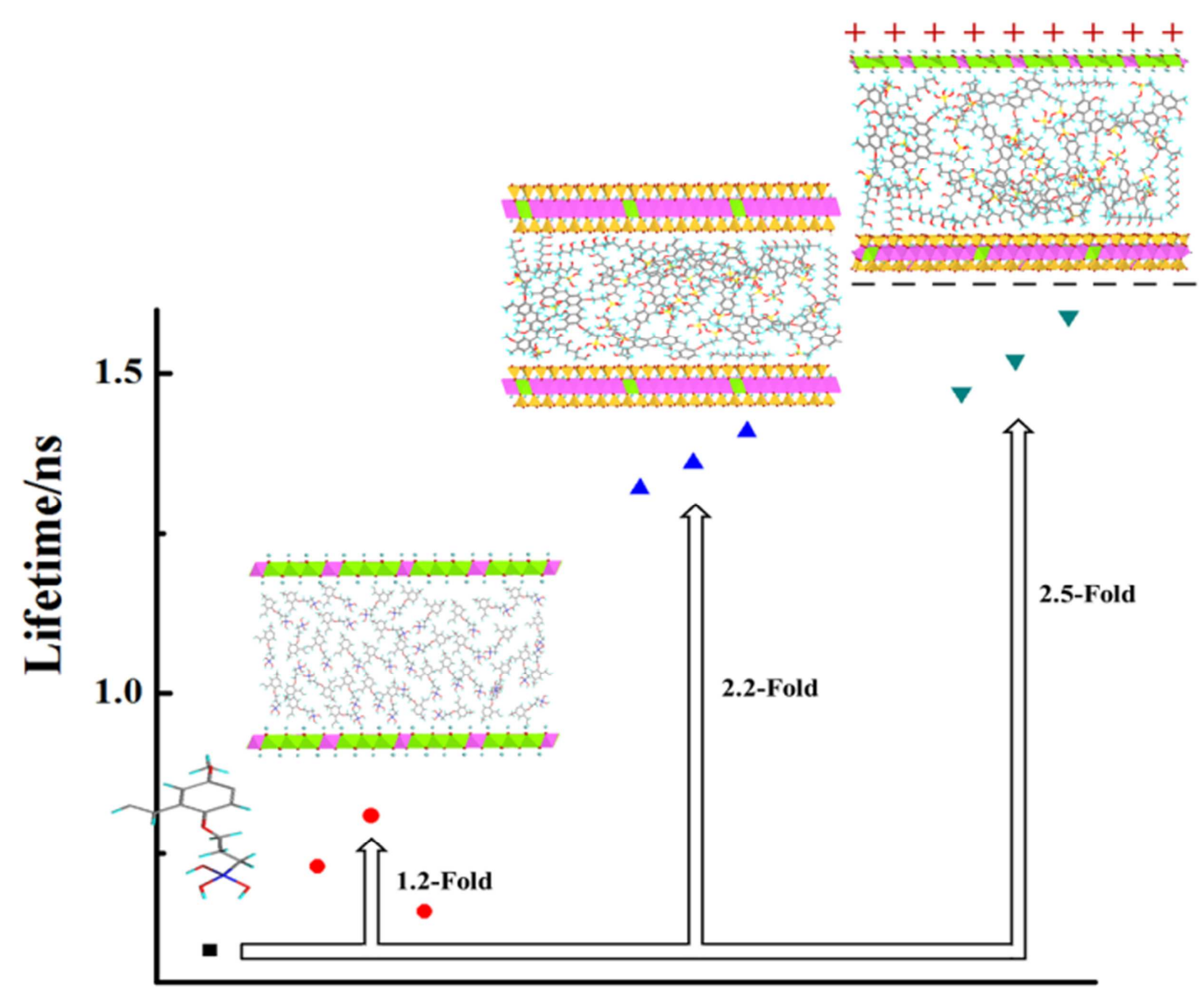

Figure 4. The comparison of APPV's lifetimes in the different states under the different environments. The black, red, blue and bottle green dots represent the lifetime of APPV, LA(n)-films, MA $(n)$-films and $\operatorname{MALA}(n)$-films, respectively. The folds stand for the different kinds of multilayer films' luminescent lifetimes compared with the pristine APPV solution.

\section{Conclusions}

To sum up, this work successfully assembled MgAl-LDHs and MMT to form an electronic microenvironment and designed the $\pi$-conjugated anionic polymer in the rigid microenvironment. Significantly, the multilayer films with ultra-prolonged lifetime were obtained, and EME is testified to be solely capable of improving the luminescent properties. According to our present work, not only can cationic chromophores be affected with better optical behavior but anionic chromophores are also effected by EME. Therefore, EME has a broad scope of application to a series of chromophores that is expected to develop in the next generation of novel luminescent multilayer films.

Acknowledgments: This work was supported partially by the National Natural Science Foundation of China (Grant No.: 40802013), the Fundamental Research Funds for the Central Universities (Grant No.: 2652015092, 2652015007). 
Author Contributions: Tianlei Wang, Meitang Liu and Hongwen Ma conceived and designed the experiments; Tianlei Wang performed the experiments; Tianlei Wang, Meitang Liu and Hongwen Ma analyzed the data; Tianlei Wang wrote the paper.

Conflicts of Interest: The authors declare no conflict of interest.

\section{References}

1. Butler, S.Z.; Hollen, S.M.; Cao, L.Y.; Cui, Y.; Gupta, J.A.; Gutiérrez, H.R.; Heinz, T.F.; Hong, S.S.; Huang, J.X.; Ismach, A.F.; et al. Progress, challenges, and opportunities in two-dimensional materials beyond graphene. ACS Nano 2013, 7, 2898-2926. [CrossRef] [PubMed]

2. Butt, F.K.; Tahir, M.; Cao, C.B.; Idrees, F.; Ahmed, R.; Khan, W.S.; Ali, Z.; Mahmood, N.; Tanveer, M.; Mahmood, A.; et al. Synthesis of novel $\mathrm{ZnV}_{2} \mathrm{O}_{4}$ hierarchical nanospheres and their applications as electrochemical supercapacitor and hydrogen storage material. ACS Appl. Mater. Interfaces 2014, 6, 13635-13641. [CrossRef] [PubMed]

3. Gentili, D.; Valle, F.; Albonetti, C.; Liscio, F.; Cavallini, M. Self-organization of functional materials in confinement. Acc. Chem. Res. 2014, 47, 2692-2699. [CrossRef] [PubMed]

4. Cavallini, M.; D’Angelo, P.; Criado, V.V.; Gentili, D.; Shehu, A.; Leonardi, F.; Milita, S.; Liscio, F.; Biscarini, F. Ambipolar multi-stripe organic field-effect transistors. Adv. Mater. 2011, 23, 5091-5097. [CrossRef] [PubMed]

5. Podsiadlo, P.; Kaushik, A.K.; Arruda, E.M.; Waas, A.M.; Shim, B.S.; Xu, J.D.; Nandivada, H.; Pumplin, B.G.; Lahann, J.; Ramamoorthy, A.; et al. Ultrastrong and stiff layered polymer nanocomposites. Science 2007, 318, 80-83. [CrossRef] [PubMed]

6. Zhuk, A.; Mirza, R.; Sukhishvili, S. Multiresponsive clay-containing layer-by-layer films. ACS Nano 2011, 5, 8790-8799. [CrossRef] [PubMed]

7. Huang, S.; Cen, X.; Peng, H.D.; Guo, S.Z.; Wang, W.Z.; Liu, T.X. Heterogeneous ultrathin films of poly(vinyl alcohol)/layered double hydroxide and montmorillonite nanosheets via layer-by-layer assembly. J. Phys. Chem. B 2009, 113, 15225-15230. [CrossRef] [PubMed]

8. Liu, Z.P.; Ma, R.Z.; Osada, M.; Iyi, N.; Ebina, Y.; Takada, K.K.; Sasaki, T. Synthesis, anion exchange, and delamination of Co-Al layered double hydroxide: Assembly of the exfoliated nanosheet/polyanion composite films and magneto-optical studies. J. Am. Chem. Soc. 2006, 128, 4872-4880. [CrossRef] [PubMed]

9. Wang, Q.; O'Hare, D. Recent advances in the synthesis and application of layered double hydroxide (LDH) nanosheets. Chem. Rev. 2011, 112, 4124-4155. [CrossRef] [PubMed]

10. Gunjakar, J.L.; Kim, T.W.; Kim, H.N.; Kim, I.Y.; Hwang, S.J. Mesoporouslayer-by-layer ordered nanohybrids of layered double hydroxide and layered metal oxide: highly active visible light photocatalysts with improved chemical stability. J. Am. Chem. Soc. 2011, 133, 14998-15007. [CrossRef] [PubMed]

11. Shu, Y.Q.; Yin, P.G.; Liang, B.L.; Wang, S.S.; Gao, L.; Wang, H.; Guo, L. Layer by layer assembly of heparin/layered double hydroxide completely renewable ultrathin films with enhanced strength and blood compatibility. J. Mater. Chem. 2012, 22, 21667-21672. [CrossRef]

12. Tanaka, T.; Nishimoto, S.; Kameshima, Y.; Miyake, M. Fabrication of layered double hydroxide/ photoresponsive dendron nanocomposite multilayer film by electrostatic layer-by-layer assembly. Mater. Lett. 2011, 65, 2315-2318. [CrossRef]

13. Dou, Y.B.; Xu, S.M.; Liu, X.X.; Han, J.B.; Yan, H.; Wei, M.; Evans, D.G.; Duan, X. Transparent, flexible films based on layered double hydroxide/cellulose acetate with excellent oxygen barrier property. Adv. Funct. Mater. 2014, 24, 514-522. [CrossRef]

14. Coiai, S.; Passaglia, E.; Pucci, A.; Ruggeri, G. Nanocomposites based on thermoplastic polymers and functional nanofiller for sensor applications. Materials 2015, 8, 3377-3427. [CrossRef]

15. Yan, D.P.; Lu, J.; Ma, J.; Wei, M.; Wang, X.R.; Evans, D.G.; Duan, X. Anionic poly(p-phenylenevinylene)/ layered double hydroxide ordered ultrathin films with multiple quantum well structure: A combined experimental and theoretical study. Langmuir 2009, 26, 7007-7014. [CrossRef] [PubMed]

16. Wang, T.L.; Liu, M.T.; Ma, H.W.; Liu, X.J.; Fu, Y.; Hu, K.R. Lifetime-ultra-prolonged luminescent multilayer thin films with electronic microenvironment. RSC Adv. 2014, 4, 40748-40752. [CrossRef]

17. Liu, M.T.; Wang, T.L.; Ma, H.W.; Fu, Y.; Hu, K.R.; Guan, C. Assembly of luminescent ordered multilayer thin-films based on oppositely-charged MMT and magnetic NiFe-LDHs nanosheets with ultra-long lifetimes. Sci. Rep. 2014, 4. [CrossRef] [PubMed] 
18. Liu, M.T.; Wang, T.L.; Ma, H.W.; Fu, Y.; Hu, K.R.; Guan, C. Layer-by-layer assembly of luminescent multilayer thin films by MMT, anionic chromophores and magnetic CoAl-LDHs nanosheets. Mater. Lett. 2015, 153, 40-43. [CrossRef]

19. Lee, W.E.; Jin, Y.J.; Park, L.S.; Kwak, G. Fluorescent actuator based on microporous conjugated polymer with intramolecular stack structure. Adv. Mater. 2012, 24, 5604-5609. [CrossRef] [PubMed]

20. Bigall, N.C.; Parak, W.J.; Dorfs, D. Fluorescent, magnetic and plasmonic-hybrid multifunctional colloidal nano objects. Nano Today 2012, 7, 282-296. [CrossRef]

21. Chung, S.J.; Kwon, K.Y.; Lee, S.W.; Jin, J.I.; Lee, C.H.; Lee, C.E.; Park, Y. Highly efficient light-emitting diodes based on an organic-soluble poly(p-phenylenevinylene) derivative carrying the electron-transporting PIED moiety. Adv. Mater. 1998, 10, 1112-1116. [CrossRef]

22. Chen, Z.K.; Meng, H.; Lai, Y.H.; Huang, W. Photoluminescent poly(p-phenylenevinylene) with an aromatic oxadiazole moiety as the side chain: Synthesis, electrochemistry, and spectroscopy study. Macromolecules 1999, 32, 4351-4358. [CrossRef]

23. Becker, H.; Spreitzer, H.; Kreuder, W.; Kluge, E.; Schenk, H.; Parker, I.; Cao, Y. Soluble PPVs with enhanced performance-A mechanistic approach. Adv. Mater. 2000, 12, 42-48. [CrossRef]

24. Chen, Z.K.; Lee, N.H.S.; Huang, W. New phenyl-substituted PPV derivatives for polymer light-emitting diodes-synthesis, characterization and structure-property relationship study. Macromolecules 2003, 36, 1009-1020. [CrossRef]

25. Jenekhe, S.A. Excited-state complexes of conjugated polymers. Adv. Mater. 1995, 7, 309-311. [CrossRef]

26. Nguyen, T.Q.; Martini, I.B.; Liu, J.; Schwartz, B.J. Controlling interchain interactions in conjugated polymers: The effects of chain morphology on exciton-exciton annihilation and aggregation in MEH-PPV films. J. Phys. Chem. B 2000, 104, 237-255. [CrossRef]

(C) 2016 by the authors; licensee MDPI, Basel, Switzerland. This article is an open access article distributed under the terms and conditions of the Creative Commons Attribution (CC-BY) license (http://creativecommons.org/licenses/by/4.0/). 